\title{
SPUTTERING FROM ENERGETIC SELF-ION CASCADES
}

by

K. L. Merkle and P. P. Pronko

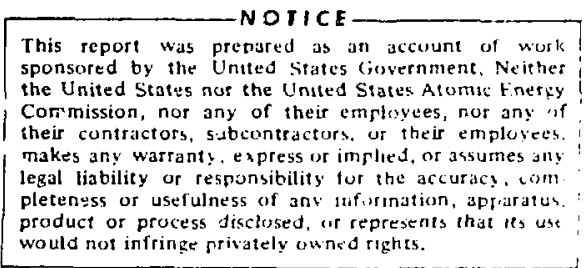

Presented at:

Surface Effects in Controlled Thermonuclear Devices and Reactors Conference, Argonne National Laboratory, Argonne, Illinois, January 10-12, 1974
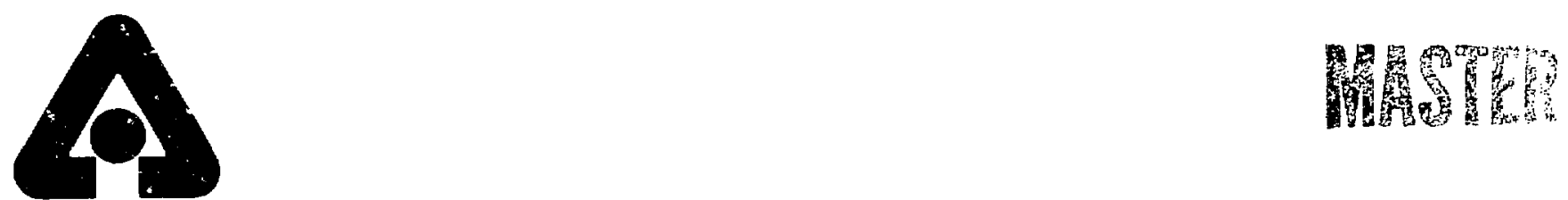

U Of C-AUA-USAEC $=$

\section{ARGONNE NATIONAL LABORATORY, ARGONNE, ILLINOIS}




\title{
SPUTTERING FROM ENERGETIC SELF-ION CASCADES*
}

K. L. Merkle and P. P. Pronko

\author{
Materials Science Division \\ Argonne National Laboratory \\ Argonne, Illinols 60439
}

January 1973

\footnotetext{
NOTICE

This report was prepared as an account of work sponsored by the United Srates riovernment. Neither the United States nor the United States Alomic Energy Cominission, nor any of their inployees, nor any of their contractors, subcontractors, or their employees. makes any warranty, express or implied, or assumes any legal liability or responsibility for the accuracy. pleteress or usefulness of any information, apparatus, product or process disclosed, or tepresents that its use would not infringe privately owned rights.
}

To be published in the proceedings of the proceedings of the Surface Effects in

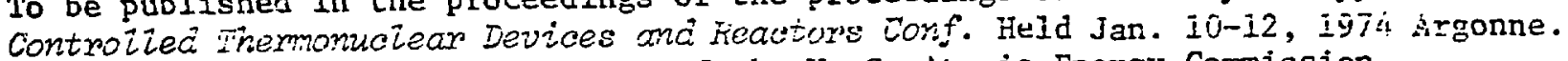
* Work performed under the auspices of the U. S. Atomic Energy Commission. 


\section{SFUTTERING FROM ENERGETIC SELF-ION CASCADES* \\ K. L. Merkle and P. P. Pronko Materials Science Division \\ Argonne National Laboratory \\ Argonne, Illinois 60439}

\section{Abstract}

The rather high primary recoil energles that occur under $14-\mathrm{MeV}$ neutron Irradiation can be simul.ted by self-ion bombardment. However, the primary Ion always starts at the entrance surface when self-ion bombardment is used. To examine the effects of rescades in which the monentum of the primary atom is directed towards an exit surface, one has to introduce a second surface that w1ll intersect the cascade in the forward direction. Self-ion transmission sputtering on very thin films has been used for this purpose. The analysis was done in situ by $\mathrm{He}^{+}$backscattering. A backsputtering ratio of 120 was observed at $540 \mathrm{keV}$. At the same energy a forward sputtering ratio of 70 is obtained for a film of $660 \AA$ in thickness. These ratios are in order of magnitude agreement with theoretical estimates, however, the results seem to indicate that collective and lattice effects play a significant role. It was observed that a tensile stress is built up in the films during irradtation, leading to flattening and rupturing along preexisting defects.

\section{Introduction}

The production of monoenergetic displacement cascades by self-ion bombardment has been used by a number of investigators to study defect structures and sputtering in metals. Defect structures have been examined using various

*Work performed under the auspices of the U. S. Atomic Energy Commission. 
forms of ion and electron microscopy (1-3) while sputtering information has been obtained from ejection patterns and material loss measurement. (4-6)

A correlation between effects from neutron induced cascades, generated by a primary knock on, with cascades from self ion irradiations, which simulate the primary knock on condition, has long been of interest. This interest arises from the fact that ion bombardment techniques afford a simple, efficient, and accurate method for producing energetic cascades in solids. Thus, ion irradiations represent a useful alternative to, and a complementary check on, cascade phenomena associated with high energy neutron collisions. For example a recent comparison of transmission electron microscopy (TEM) data obtained from self ion bombardients with data resulting from $14-\mathrm{MeV}$ neutron irradiation has demonstrated good quantitative agriement.(7)

Recently sputtering from 14-MeV neutrons has recelved considerable attention. (8-11) In a number of such experiments the total sputtering yield was found to be significantly greater than that predicted by theory. In addition to single atom sputtering and the emission of small clusters of atums, ejection of macroscopic particles has also been reported. ( 8 )

Since most of the displacements produced by $14-\mathrm{MeV}$ neutrons occur in very energetic cascades ( $100 \mathrm{keV}$ ), it is of crucial importance to know the sputtering mechanisms for such cascades when attempting to understand high energy neutron ejection.

The purpose of the present investigation was to determine whether those aspects of $14-\mathrm{MeV}$ neutron sputtering relating to the ejection from energetic displacement cascades could be studied by means of self ion bombardment. of particular interest in this connection are those cascades that lead to very high sputtering ratios. In making these comparisons an important difference between the $14-\mathrm{MeV}$ neutron recoil cascades and self ion cascades is that self ion cascades always start at the entrance surface. Recoil cascades, 
however, w11l be Initiated with equal probability at any depth from the surface end, most importantly, the recoli momentum of the primary particle can also be directed towards a surface. In the case of the self-ion cascade, the primary ion is initialy always going into the entrance surface. Results from such experiments cannot, therefore, be directly related to neutron sputtering ylelds.

In the present experiment we were interested in duplicating the situation where a primary recoll initially moves towards a surface at a distance comparable to the mean damage depth. This can be done by self-ion bombardment of thin fllms where the transmission sputtering rate is measured from filins whose thickness is comparable to the mean damage depth. As seen in Fig. 1, displacement .cascades of some hundreds of $\mathrm{keV}$ split inl, subcascades. Each subcascade that intersects a surface can give rise to sputtering. Basically any atom that arrives at the surface with a kinetic energy greater than the surface binding energy will be sputtered. In a very energetic cascade more than one subcascade can be intersected by the surface. The number of sputtered atoms that are ejected from a subcascade will depend strongly on the position of the surface relative to the center of the subcascade. If the energy density in the core of a subcascade is very high, one can expect the influence of collective effects. As Anderson and Bay(12) have shown by their molecular ton experiment, an increase is the cascade density leads to a more than linear increase in the sputtering rate. In the case of very energetic cascades, where several subcascades are formed, there also is a finite probability of overlap between subcascades. An increase in the subcascade density results if two subcascades overlap, whereupon consequences similar to those observed in the Anderson-Bay experiment(12) can be expected. The core of a subcascade may have a very high displacement 
density, in which case a large fraction of the atoms will be involved in the displacement process, allowing it perhaps to be described by a thermel spike model. In this case, a relatively large amount of material could be ejected if the core of the subcascade is intersected by an external surface.

\section{Experimental}

\section{Specimen Preparation}

Thin monocrystalline films of Au were grown on (001) cleavage surfaces of rocksalt at $350^{\circ} \mathrm{C}$. During evaporation the ion-pumped evaporator was at a pressure of approximately $10^{-7}$ Torr. Single-crystalline films of (O0I) orientation were obcained in this way. Subsequently the films were annealed in air at $350^{\circ} \mathrm{C}$ for 30 rinutes. Film thickness was determined by gravimetry. After dissolving the rocksalt in water the specimens were mounted on staridard TEI copper specimen grids.

\section{Transmission Sputtering}

The grilis were transferred to a UHV scattering chamber which was attached to a $300 \mathrm{kV}$ heavy ion accelerator. As shown in Fig. 2, the film was mounted in an Al holder and the material sputtered in transmission was collected by an AI catcher. Several Al collectors could be sequentially rotated into position. The specimen holder could also be moved out of the path of the beam so that the Al collector could be examined in situ by $\mathrm{He}^{+}$backacattering. The self ion transmission sputtering was performed with a doubly charged $\mathrm{Au}^{++}$beam of $540 \mathrm{keV}$. This energy, although nearly twice the maximum for elastic 14-MeV neutron colIisions in $\mathrm{Au}$, was used because thinner specimens were not available and because the same basic ejection mechanisms are expected in the region of subcascade formation. The Au-beam was $3.2 \mathrm{~mm}$ in diameter at the specimen and was swept in two dimensions to achieve uniformity of irradiation. The dose measurement was 
obtained by means of a Faraday cage that intercepted an annular fraction of the beam in front of the target. During irradiation the pressure in the target chamber was on the order of $5 \times 10^{-8}$ Torr. Backscattering

The $f 1 l m$, as well as the collectors were analyzed in situ by means of $270 \mathrm{keV} \mathrm{He}{ }^{+}$Rutherford backscattering at $\theta=150^{\circ}$. One can determine, by thts wethod, the loss of material from the fllm(13) aid also the amount of material that has accumulated on the collectors. These, data result, therefore, in a direct measure of both the total yield (from specimen thickness reduction) and the forward yield, as obserred on the collectors.

When analysing the film a beam of 1 Im in diameter was used while a swept $3.2 \mathrm{~mm}$ iiameter beam was used to observe the sputtered Au on the collector. A laser bean whose path coincided with the ion beam greatly facilitated line up of target and collector. Figure 3 shows $a$ composit of several backscattering spectra. The channel numbers given on the abscissa can, in the usual manner, be translated into an energy or depth scale. Ine solid line represents the scattered intensity from $\mathrm{Au}$ that has been sputtered and collected on the $\mathrm{Al}$ catcher. The open points indicate the background from the Al substrate. The position of the Au-peak coincides with the position at half maximum of. the front edge of the sfectrum from a gold film. The width at half maximum of the sputtered gold peak also coincides with the width of the edge of the gold film. This Indicates that the sputtered Au is located directly at the surface of the Al catcher. If a significant fraction of the gold would have been implanted to a finite depth in the $A j$ or If particles of a finite thickness, comparable to the film thickness would have been emitted, the sputtered Au spectrum would extend to lower energles. 
For calibration purposes a $50 \mathrm{keV}_{\mathrm{Au}}^{+}$implant was inade into one of the Al collectors. The backscattering spectrum from this implant is also shown in Fig. 3. It can be seen that the most probable position of the implanted $\mathrm{Au}$ is at some finite depth in the Al. Range straggling is also seen from the increase in width of the spectrum. The calibration factor for the amount of Au present was derived from the integrated number of counts in each peak ani from the known implant dose. This calibration factor was in good agreement, with the cne derived from the Rutherford backscattering cross section and the scatcering geometry.

\section{Results}

\section{Transmission Yields}

The self ion transmission sputtering yield as a function of fluence is plotted in Fig. 4 for an Au film of $656 \AA$ thickness. In determining the yield from the amount of hu present on the Al collector within the $3.2 \mathrm{~mm}$ diameter area samplad by the $\mathrm{He}^{+}$beam, we have assumed that the amount of sputtered gold Is proportional to $\cos \psi$, where $\psi$ is the angle to the surface normal. A sticking coefficient of 1 for $A u$ on $A 1$ has also been assumed. It can be seen from Fig. 4 that the transmission yield increases linearly with dose. The straight line in Fig. 4 corresponds to 69 atoms sputtered in transmission for each incoming ion.

\section{Effects of Radiation on Film Structure}

Similar to the backsputtering thickness measurement of film on a substrate(13) one would expect to be able to determine the total sputtering (forvard and backward) from examining the Rutherford backscattering spectra of non-backed films. For uniform sputtering one expects a reduction in the width of the filn spectrum with increasing $\mathrm{Au}^{++}$dose. In contrast to this we see in Fig. 5 , after an incubation period corresponding to a dose of approximately $4 \times 10^{13} \mathrm{Au}^{+\frac{1+}{4} / \mathrm{cm}^{2}}$, 
a reduction in the height and also a widening rather than a decrease in width of the spectrum. A decrease in height is expected if holes are formed in the f1lm. Figure 6 shows what actually has happened to the film. Rather large hnles have appeared and in some instances the film has partially folded over. The latter would account for the apparent increase in width of the spectrum. The most striking feature is that the film has otherwise become completely flat during the self ion irradiation. The upper right hand corner of the film has not been Irradiated. Apparently during irradiation a tensile stress has built up in the film straightening out the wrinkles and finally tearing the film apart. TEM observation clearly indicates that mechanical deformation is involved in forming the gaps. It can also be seen by TEM that the tears start at some weak points in the film. These weak points are mostly linear reyions, characterized by a reduced film thickness and the presence of small holes. Those linear defects are introduced during evaporation of the films and come from the effects of cleavage steps or scratches in the rocksalt substrate. It is possible that the tears could be avolded if these preexisting defects could be eliminated.

\section{Backward Sputtering Yield}

To datermine the backward sputtering yield we sputtered a $1200 \AA$ thick film that was still attached to the rocksalt substrate. In this case the film did not form large holes as in the unsupported film. The reduction in thickness is clearly seen in the He-backscattering spectra after various irradiation doses as shown in Fig. 7. The backward sputtering ratio derived from this reduction in thickness is $\mathrm{s} \approx 120$.

\section{Comparison witli Theory}

The theory of sputtering from amourphous targets has been given comprehensive treatment by $P$. Sigmund. (14) The theory agrees rather well with a 
number of sputtering measurements on polycrystalline targets $(12,14)$ and it is also expected to give a good indication of the total sfuttering yield for a beam incident under random direction on a single crystaline target. According to ref (14) the sputtering yield $S$ as a function of energy $E$ is given by

$$
S(E)=\Lambda F(E)
$$

where under the: present condit:ons $\Lambda:=0.187 \AA^{-2}$. In first approximation the function $F(E)$ is given by

$$
F(E)=\frac{\nu(E)}{\left\langle\Delta \mathrm{x}^{2}\right\rangle / 2} \frac{1}{\sqrt{2} \pi} \mathrm{e}^{-\xi^{2} / 2}
$$

with $\xi=-\langle x\rangle /\left\langle\Delta x^{2}>1 / 2\right.$ for backward sputtering and $\xi=\left(d-\langle x\rangle j /<\Delta x^{2}\right\rangle 1 / 2$ for transmission sputtering. Here $v(E)$.. the energy that is dissipated in nuclear collisions, $d$ is the film thickness and $\langle x\rangle$ and $\left\langle\Delta x^{2}\right\rangle$ are moments over the deposited energies given in ref (15) for various power law cross sections. With $\mathrm{m}=1 / 2$ (see ref.15) we get $\langle\mathrm{x}\rangle=378 \AA$ and $\left\langle\Delta \mathrm{x}^{2}\right\rangle=1 / 2=233 \AA$. If we assume $\nu(E)=E$ we find $S_{b}=50$ and $s_{t}=85$ for the backward and transmission sputtering ratio respectively. Taking into account that about $1 / 3$ of the energy is lost in electronic exitaticns(16) the above yields are reduced to $s_{b}=33$ and $s_{t}=56$. Sigmund et al. (14) have also considered the influence of electronic stopping on the moments $\langle x\rangle$ and $\left\langle\Delta x^{2}\right\rangle$. For this case one gets $\langle x\rangle=273 \AA$ and $\left\langle\Delta x^{2}\right\rangle 1 / 2=150 \AA$ which gives $s_{b}=32$ and $s_{t}=5.5$ for the backward and forward sputtering ratics respectively.

In comparing these theoretical values with our experimental resilts we find that the observed back sputtering ratio is a factor of 2 to 4 tigher than predicted by theory. Similar increases above the thenretical yields have previously been found for $\mathrm{Ag}(12)$ The values for the transmission ratios are In rather good agreement with the experiment for the case where the influence of electronic losses on the deposited energy distribution is neglected. The rather low yield $s_{t}=6.5$ which is obtained when electronic losses are taken 
Into account illustrates the strong dependence of the transmission yield on average damage depth or film thickness. TEM work has indicated that the size of the cascade is larger than indicated by the rangom theory. (18) This increased size is probably due to channeling effects. Larger yields at the present ti:ickness and a smaller thickress dependence of the transmission sputtaring yields would be expected from channeling effects.

\section{Conclusions}

1. The observed backsputtering ratios from energetic self-ion cascades are somewhat higher than prediced by the random cascade theory. However, the deviations are within a factor of 4 of the theoretical value and are probably due to collective etfects.

2. Transmission sputtering yields are in good agreement with the simple theory, however, thickness dependence measurements are essential for a more quantitative comparison with theory.

3. Tensile stresses in the filns are 'uilt up during self ion irradiation, leading to film flattening and rupture along preexisting defects. The loss of interstitials from the interior of the film due to diffusion to the surface and sputtering, together with the spontaneous formation of vacancy clusters in energetic cascades causes internal stresses that lead to this effect. This "stretching effect" is opfosite to the "dimpling" effect that has been observed in Si under He bombardment. (19)

4. The ejection of macroscopic particles has not been observed in the present experiment. The total material loss of the target as estimated from the backscattering spectra is of the same order as the sum of the transinission and backscattering yields.

5. The method introduced here for measuring thin film transmission yields seems to be well suited to more detailed studies. Within certain limits it Is possible to study the thickness and energy dependence of self-ion sputtering. 


\section{$\underline{\text { References }}$}

1. D. N. Seldman, J. Phys. F, 3, (1973).

2. M. Wilkens in "Vacancies and Interstitials in Metals", North Holland, Amsterdam, p.485, (1969).

3. B. L. Eyre, J. Phys. F. 3, 422 (1973).

4. M. W. Thompson, Defects and Radiation Damage In Meta?s, Cambridge University Press (1969).

5. M. Kaminsky, Atomic and Ionic Impact Thenomena on Metal Sur:Faces, Springer Verl., Berlin - Academic Press, New York (1965).

6. R. Behrisch, Ergeb. Exact. Naturw. 35, 295 (1964).

7. K. L. Merkle, Trans. Am. Nucl. Soc. 17, 138 (1973).

8. M. Kaminsing and S. K. Das, This conference.

9. T. H. Blewitt, M. A. Kirk, A. C. Klank, R. Malewicki and T. L. Scott, This conference.

10. M. T, Robinson, This conference.

11. M. Guinan, This conference.

12. H. H. Anderson and H. L. Bay, Rad. Eff. 19,139 (1973).

13. R. Weissmann and R. Behrisch, Rad. Eff. 19, 69 (1973).

14. P. Sigmund, Phys. Rev. 184, 383 (1969).

15. K. B. Winterbon, P. Sigmund and J. B. Sanders, Mat. Fys. Medd. Dan. Vid. Selsk $\underline{37}, 14$ (1970).

16. J. Lindhard, V. Nielsen, M. Scharff and P. V. Thomsen, Mat. Fys. Medd, Dan. Vid. Selsk $\underline{33}$, No. 10 (1963).

17. P. Sigmund, M. T. Matthies and D. C. Philips, Rad, Eff. 11, 39 (1971).

18. K. L. Merkle, Bu11. Am. Phys. Soc. 18, 479 (1973).

19. R. L. Meek, W. M. Gibson and J. P. F. Sellschop, Rad. Eff. 11, 139 (1971). 


\section{Eigure Captions}

Fig. 1. TEM 1.mages of $540 \mathrm{keV}$ self-ion displacement cascades in Au, exhibiting formation of subcascades. Each group of spots was produced by a single lon. The black spots represent vacancy clusters that are formed in the depleted zone of subcascades.

Fig. 2. Specimen geometry for thin film sputtering in forward and back directions.

FIg. 3. Compostte of information obtainable from backscattering measurements.

Fig. 4. Transmission sputtering yields as a.function of bombardment fluence.

Fig. 5. He $e^{+}$backscattering spectra from an Au foil mounted on a Cu grid for various $\mathrm{Au}^{++}$bombardment fluences.

Fig. 6. Optical micrograph of $\mathrm{Au}$ f $11 \mathrm{~m}$ after irradiation to a total fluence of $2.8 \times 10^{14} \mathrm{Au}^{++} / \mathrm{cm}^{2}$ at $540 \mathrm{keV}$. Magnification $70 \mathrm{x}$.

Fig. 7. He ${ }^{+}$backscattering spectra of Au film on NaCl substrate after various fluences of $\mathrm{Au}^{++}$at $540 \mathrm{keV}$. 


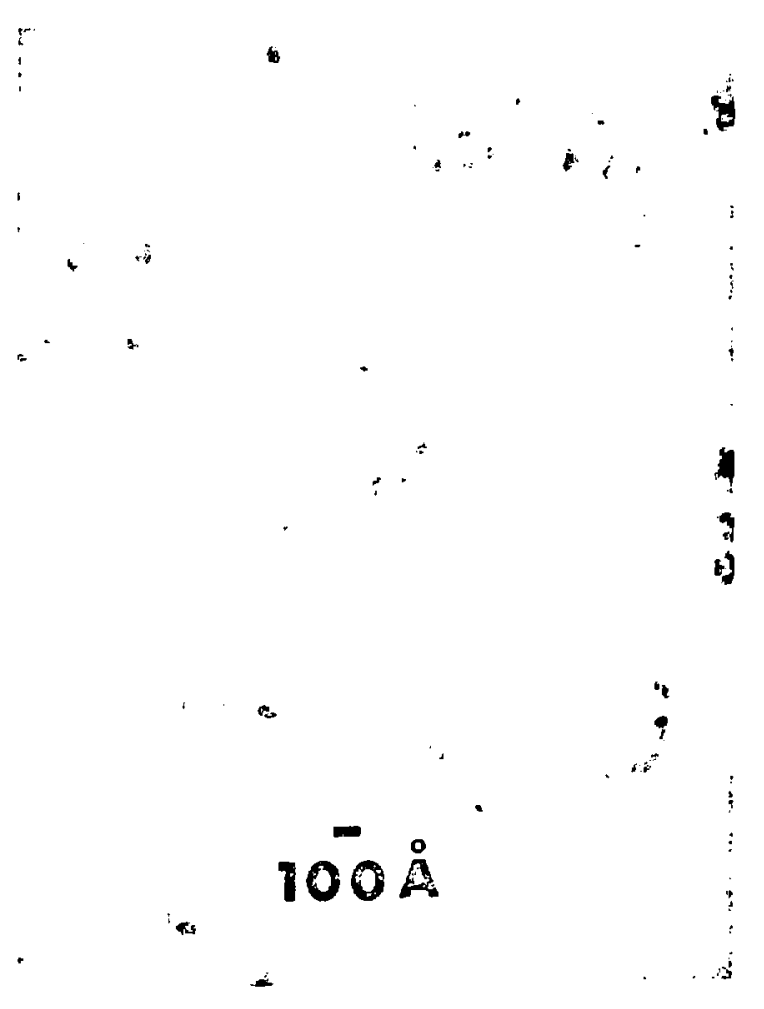

Fig. 1 
TRANSMISSION SPUTTERING

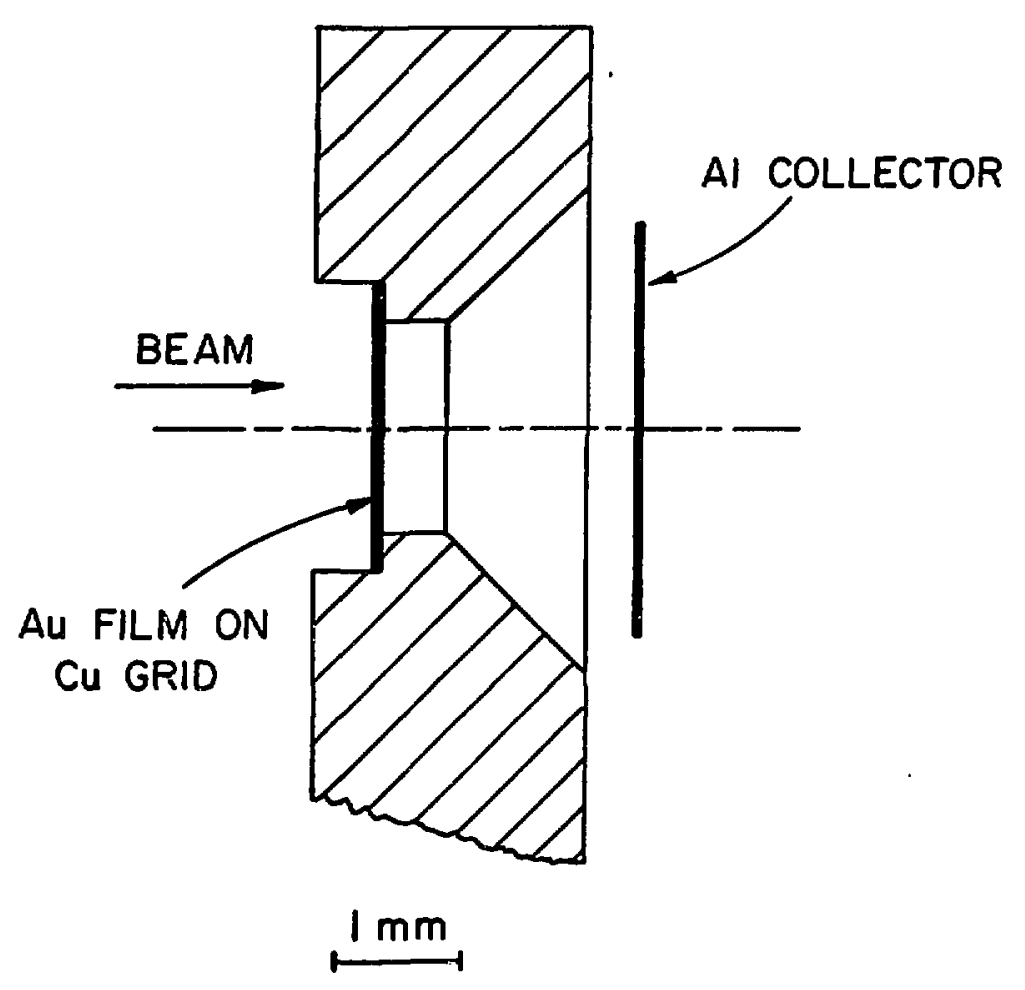

Fig. 2 


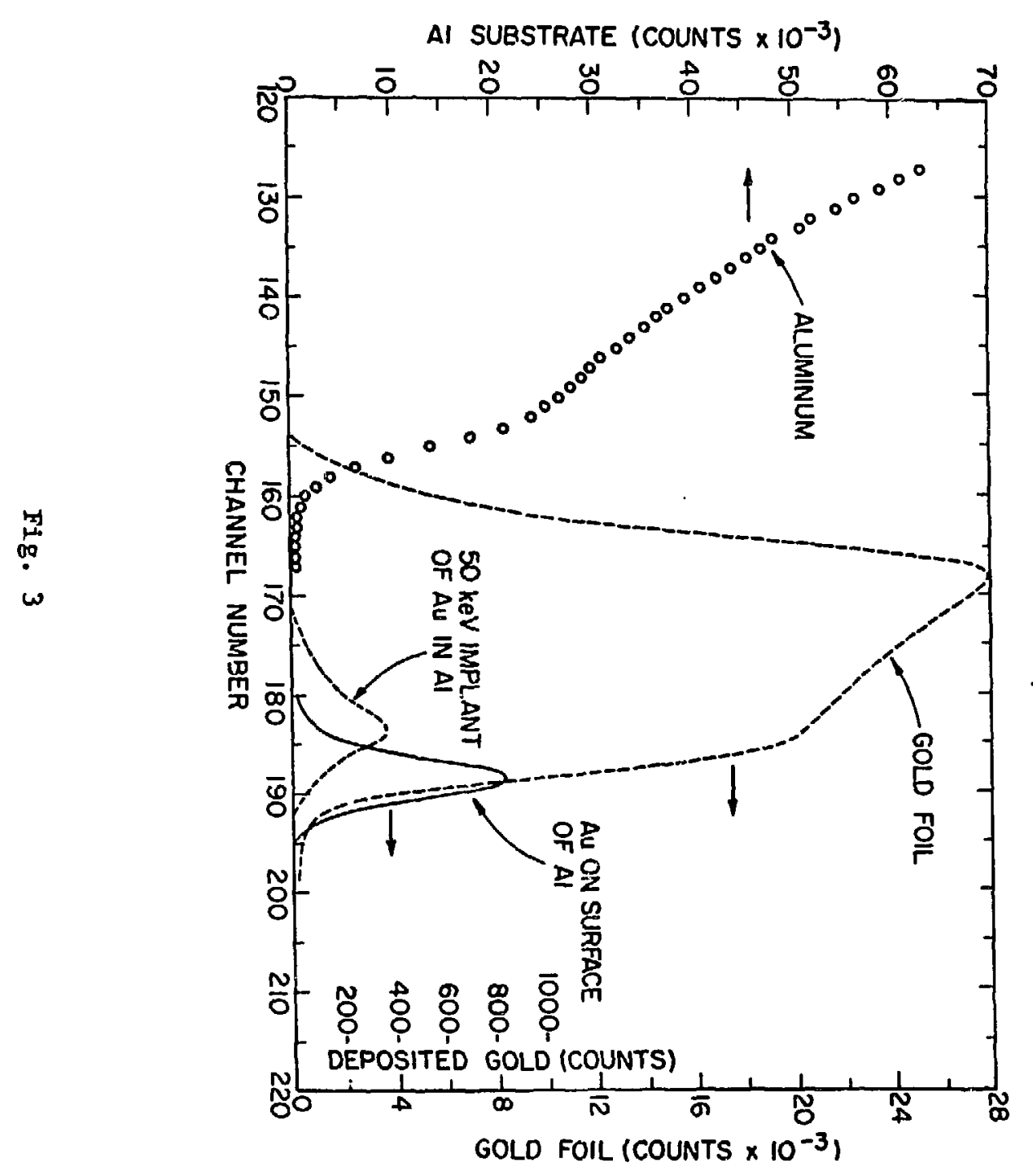




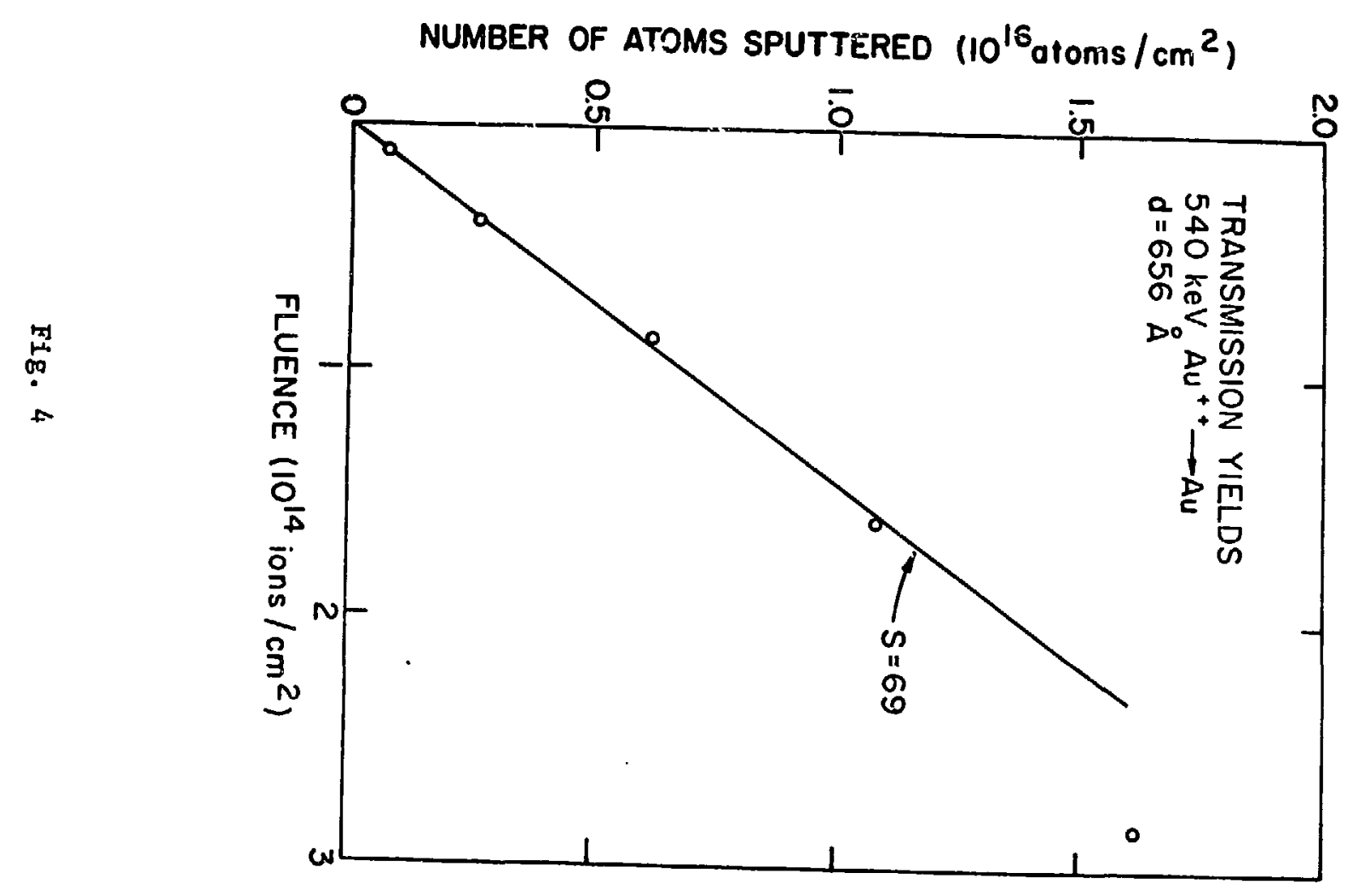




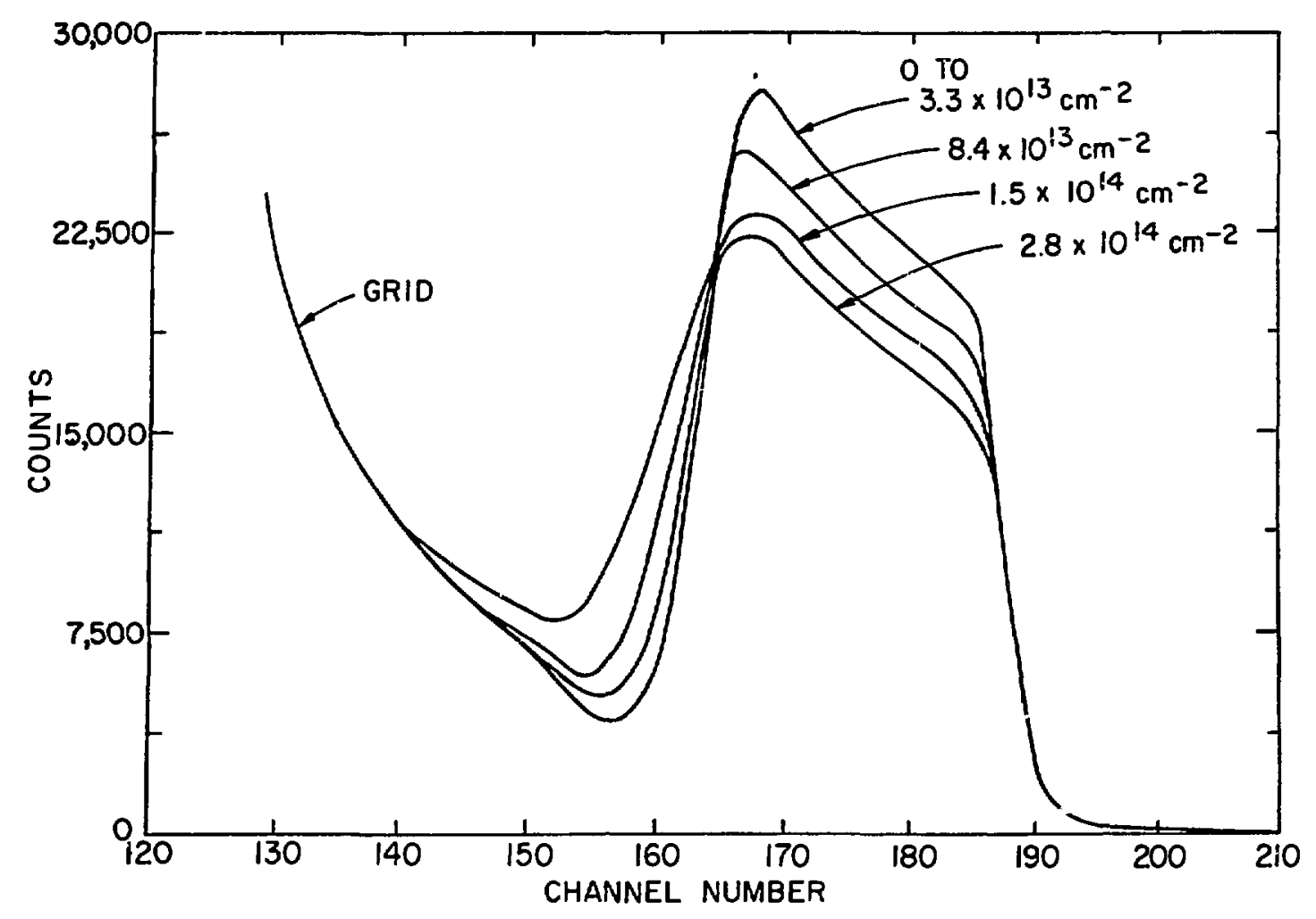

Fig. 5 


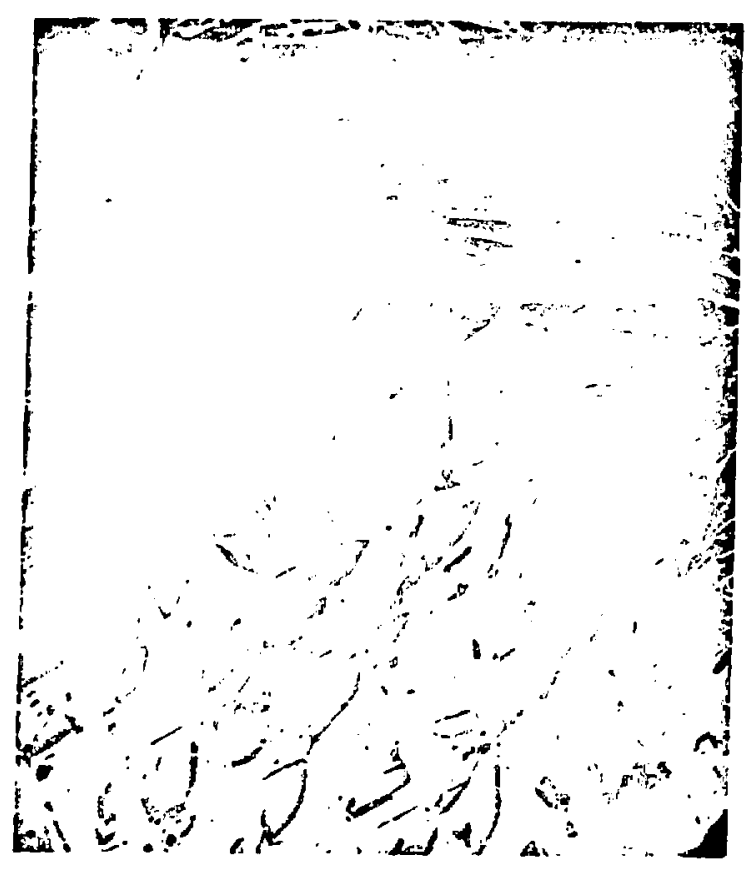

Fig. 6 


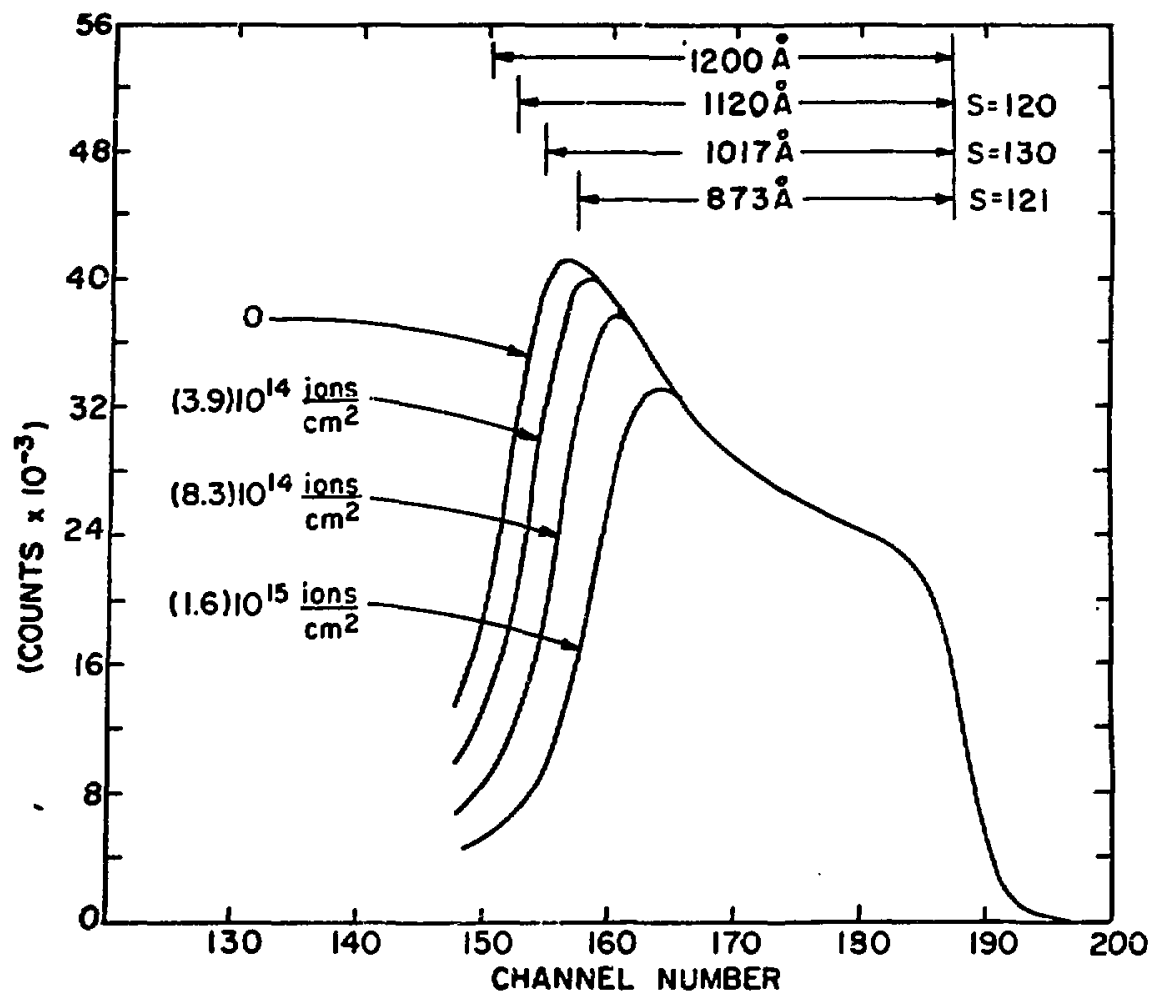

Fig. 7 